\title{
Concepciones sobre afectividad en docentes que trabajan en aulas hospitalarias en Chile
}

SYLVIA CONTRERAS SALINAS*

Universidad de Santiago de Chile- Chile

FLOR ROMERO ORMEÑO**

Universidad de Santiago de Chile- Chile

Recibido el 21-11-18; primera evaluación el 05-12-19; segunda evaluación el 08-02-20; aceptado el 19-02-20

\section{RESUMEN}

Este artículo describe las concepciones de afectividad en los discursos de docentes que trabajan en un aula hospitalaria perteneciente a un hospital público de la Región Metropolitana en Chile, considerándola como una experiencia multidimensional, desde la cual se configura la práctica docente. La metodología y procedimientos corresponden a un enfoque cualitativo, específicamente, un estudio de caso. Para esto, se entrevistó a la totalidad del equipo docente que atiende a nińos diagnosticados con cáncer. Los principales hallazgos indican que la categoría afectividad no es visualizada como un fenómeno que sea teorizado y recreado en el discurso de los docentes entrevistados. Finalmente, se concluye señalando que es necesario avanzar en valorar y esclarecer la afectividad puesta en juego en el acto educativo.

Palabras clave: afectividad, docentes, pedagogía hospitalaria

\footnotetext{
* Doctora en Pedagogía de la diversidad sociocultural por la Universidad Complutense de Madrid. Magister en Educación con Mención en Currículo y Evaluación. Socióloga y profesora de Educación diferencial. Docente e investigadora en distintas universidades chilenas, entre ellas, la Universidad de Santiago de Chile. En 2015, se adjudica un proyecto Fondecyt (Fondo Nacional de Desarrollo Científico y Tecnológico) de Iniciación titulado: Trayectorias educativas y saberes socialmente producidos que despliegan mujeres inmigrantes en los procesos de inclusión en la Región Metropolitana de Chile. Correo electrónico: sylvia.contreras.s@usach.cl

** Egresada de técnico en Educación Parvularia del Colegio Politécnico Santa Ana. Licenciada en Educación. Docente de Educación general básica por la Universidad de Santiago de Chile con mención en Lenguaje, Comunicación y comprensión del Medio Social. Docente de Lenguaje en una escuela de la comuna de Santa Cruz, VI Región, Chile. Correo electrónico: flor.ormeno@gmail.com
} 
Conceptions about affectivity in teachers working in hospital classrooms in Chile

\section{Abstract}

This article describes the conceptions of affectivity in the speeches of teachers who work in a hospital classroom belonging to a public hospital in the Metropolitan Region in Chile are explored, considering it as a multidimensional experience, from which the teaching practice is configured. The methodology and procedures correspond to a qualitative approach, specifically, a case study. For this, the whole teaching team that attends children diagnosed with cancer was interviewed. The main findings indicate that the category affectivity is not visualized as a phenomenon that is theorized and recreated in the discourse of the teachers interviewed. Finally, it is concluded that it is necessary to advance in valuing and clarifying the affectivity put into play in the educational act.

Keywords: affectivity, teachers, hospital Pedagogy

Concepçóes sobre afetividade em professores que trabalham em salas de aula hospitalares no Chile

\section{RESUMO}

Este artigo descreve as concepçóes de afetividade nos discursos de professores que trabalham em uma sala de aula de um hospital público de um hospital público da Região Metropolitana do Chile, considerando-a como uma experiência multidimensional, a partir da qual se configura a prática docente. A metodologia e os procedimentos correspondem a uma abordagem qualitativa, especificamente, um estudo de caso. Para isso, toda a equipe de ensino que atende crianças diagnosticadas com câncer foi entrevistada. Os principais achados indicam que a categoria afetividade não é visualizada como um fenômeno teorizado e recriado no discurso dos professores entrevistados. Por fim, conclui-se que é necessário avançar na valorização e clarificação da afetividade posta em ação no ato educativo.

Palavras-chave: afetividade, professores, pedagogia hospitalar 


\section{INTRODUCCIÓN}

Considerando que en la actualidad se han ido registrando cambios en la educación formal y no formal, particularmente en y desde los desafíos de educación inclusiva, es innegable su efecto en las nuevas responsabilidades de los docentes, conformándose especificidades que generan demandas en relación con la formación inicial como al desarrollo profesional docente, que comprometen su identidad profesional. Además, se encuentra tensionada por la necesidad de atender la diversidad en y, desde una educación humanizada, que atienda a la condición de vulnerabilidad (Carabantes y Contreras, 2020; Nunes, 2019), especialmente en el contexto de las aulas hospitalarias.

\subsection{Derecho a la educación: aulas hospitalarias y trabajo docente}

La Declaración Universal de Derechos Humanos señala en el artículo 26 que toda persona tendrá derecho a educarse. Agrega que la enseñanza «tendrá por objeto el pleno desarrollo de la personalidad humana y el fortalecimiento del respeto a los derechos humanos y a las libertades fundamentales» (Organización de las Naciones Unidas, 2015, p.8).

Particularmente, existen niños, niñas, adolescentes y jóvenes que, por razones de salud, deben permanecer temporalmente hospitalizados, generando una situación de desventaja dentro del sistema educativo, con posibles alteraciones emocionales. Es así que se requiere una respuesta educativa que garantice la continuidad del proceso educativo contemplando las necesidades de tipo afectivo (Garrido, 2016).

Una de las respuestas frente a esta situación es la pedagogía hospitalaria, la cual constituye una modalidad educativa cuyo principal objetivo es hacer efectivo el derecho a la educación de los niños, niñas y jóvenes hospitalizados, previniendo la marginación sociocultural y del sistema educativo (Arredondo, 2014; Ministerio de Educación, 2003). En otras palabras, se busca que los estudiantes se reintegren a la escolaridad, adaptando las clases a las distintas formas de aprender, respetando ritmos, capacidades, motivaciones e intereses particulares (OREALC/Unesco, 2007). El docente debe generar una relación con el estudiante para que exprese sus afectaciones, emociones y pensamientos para disminuir su miedo y ansiedad, generadas por habitar en un espacio adverso.

En otras palabras, la pedagogía hospitalaria se define como una disciplina que estudia e integra actuaciones educativas y psicoeducativas dirigidas a personas que padecen diversas enfermedades y a sus familias, intentando dar respuesta a sus necesidades biopsicosociales, para desarrollar sus potencialidades 
y mejorar su calidad de vida (Molina, 2017). Para esto, se diseñan e implementan una serie de actividades con la participación de profesionales del área de la salud y la educación, cobrando sentido el trabajo multidisciplinario entre equipos (Arredondo, 2014).

A nivel regional, la pedagogía hospitalaria se reconoce a partir de la Declaración de los Derechos del Niño, Niña o Joven Hospitalizado o en Tratamiento, de América Latina y El Caribe en el Ámbito de la Educación, proclamada en 2009 y validada oficialmente en 2013. Este documento se compone de doce artículos que contemplan todos los niveles de escolaridad, incluyendo la modalidad diferencial y especial, además de las respectivas normativas para los recintos médicos y escolares (Red Latinoamericana y del Caribe [Redlaceh], 2009).

En este contexto, el rol de los docentes que trabajan en las aulas hospitalarias resulta relevante, sin embargo, tampoco se encuentra ajeno a tensiones y desafíos, así como al hecho que la definición de su labor se configura en los profundos cambios producidos en el trabajo docente, fruto del impacto de los procesos de precarización laboral, el incremento de las tareas y responsabilidades asignadas, el aumento de las problemáticas de salud mental, así como una suerte de crisis de sentido de la práctica docente.

Se podría pensar que lo descrito es más agudo en el caso de las escuelas o aulas hospitalarias, donde los docentes deben tener presente las diversas situaciones o afecciones de cada estudiante, más aún si se atiende en las modalidades de domicilio, aulas multigrado o atención "cama a cama» en el hospital. Esto ocurre en un contexto, donde no siempre la labor que los docentes desarrollan es bien valorada, especialmente cuando el personal de salud muestra una actitud pasiva y desinteresada frente al aula que funciona en el hospital (Sánchez y López, 2016).

Conjuntamente, el trabajo del profesor en estas modalidades educativas adquiere un carácter preminentemente relacional, afectivo y emocional, lo que lleva a que el discurso se organice en torno a la formación de vínculos y a una pedagogía desde los afectos (Bustos y Cornejo, 2014), basada en una real implicación (Contreras y Ramírez, 2014). Es decir, una educación de la fragilidad y finitud cobra realce (Mèlich, 2003), una educación que «no esquiva lo lábil de la existencia humana, sino más bien encuentra sentido en la acción de ahondar en su vulnerabilidad, en lo agrietado del existir, en sus claros oscuros» (Contreras, 2012, p.80).

En este contexto, se apela a una pedagogía del tacto, que tiene como principios la responsabilidad, la oportunidad, la ocasión y el arte de la improvisación: Una pedagogía que trata a cada persona como un ser singular, único 
e irrepetible (Bárcena, Larrosa y Mèlich, 2006). En otras palabras, se aspira a una pedagogía desde la ética, donde se hable acerca de las consecuencias que tienen nuestras acciones y el valor de las mismas, referido tanto a la alteridad responsable (se es responsable siempre de algo o alguien) como a la corresponsabilidad (ejecución de las acciones). Esperando que la labor docente promueva una ética del cuidado en que los docentes sean «capaces de adaptarse a diferentes situaciones, de trabajar adecuadamente el tema afectivo y emocional» (Reyes-Laredo, Martín-Rubio, y Daza-Navarro, 2018, p. 10).

\section{MarCo TEÓRICO}

$\mathrm{Si}$ aceptamos la complejidad e importancia del quehacer educativo en las aulas hospitalarias, la perspectiva fenomenológica nos permite develar las experiencias que se constituyen en ese espacio educativo con la posibilidad de re-significar lo educativo, especialmente en y desde el fenómeno de la afectividad. En otras palabras, "tener un "modo de ver", por medio del cual se ve lo puesto entre paréntesis, lo suspendido, la epojé del mundo naturalmente educativo» (Jiménez y Vázquez, 2017, p. 255), posibilitando nuevas significaciones ligadas a las concepciones que construyen los docentes. Elementos que a continuación desarrollamos.

\subsection{Experiencia afectiva: implicancias y alcances desde la perspectiva fenomenológica}

Tal como lo plantean algunos investigadores, en el marco de las políticas públicas, la labor docente se ve enfrentada a contradicciones sustantivas que afectan también a quienes la desempeñan en las aulas hospitalarias, evidenciándose, que dicho trabajo se encuentra tensionado por versiones «identitarias» que apuntan a la dimensión afectiva y emocional de su labor (Soto et al., 2016).

En este sentido, Ascorra (2009) refiere que el trabajo docente se identifica como inmaterial por realizarse predominantemente en la dimensión afectiva. Sin embargo, situar su desempeño en la categoría de docente afectivo podría tender a confundir temas como la responsabilidad profesional, puesto que contribuye a invisibilizar los aspectos estructurales y materiales que los afectan, debido a que las soluciones planteadas frente a las dificultades quedarían principalmente en el plano afectivo (Soto et al., 2016). Desde aquí, intentamos relevar que la afectividad no solo da cuenta de la afectación del sujeto en y desde el mundo, sino que es inherente al dominio moral, ético y político (Bortolan, 2017; Slaby, 2016; Elpidorou y Freeman, 2015; Freeman 
y Elpidorou, 2015), dando cuenta, incluso, de una afectividad normativa (Núñez, Núñez e Irisarri, 2017).

En consonancia, la afectividad adopta una función reveladora y estructuradora del contexto y las posibilidades que constituye el «ser en el mundo». Por tanto, desde la experiencia afectiva se revela el significado de la compleja existencia, debido a que la afectación que se muestra en su habitar le es constitutiva (Heidegger, 1974). A partir de aquí, el sentido de la práctica de la afectividad se define, ontológicamente, como una experiencia de «sí mismo» que implica estar afectado como condición ontológica que encuentra su fundamento en el mundo, específicamente, en el modo de la pasividad situada en el «estar-en-la-realidad-del-mundo» (Ballen, 2012).

Asimismo, es importante considerar que la noción de afectividad hace referencia a la pluralidad de sentimientos que se pueden experimentar, y abarca tanto estados intencionales como no intencionales, emociones efímeras y de larga duración, estados de ánimo, rasgos de carácter e incluso intuiciones, destacando desde la perspectiva fenomenológica que la experiencia afectiva está conectada además con estados cognitivos como creencias y juicios, así como también a deseos y acciones. En otras palabras, se encontraría intrínsecamente relacionada a lo encarnado y situado del devenir humano (Bortolan, 2017).

En este marco, cobra relevancia profundizar en la experiencia afectiva, la que adopta diversos matices en los distintos escenarios, y en las relaciones entre los agentes participantes, particularmente en las aulas hospitalarias, en especial, cuando se plantea comprenderla asociada a dinámicas transpersonales intensas, que se vinculan a una multiplicidad de "otros» anclados a diversos entornos. Es decir, una afectividad situada y material.

Admiten que tanto la estructura de la experiencia afectiva como su conexión con otras dimensiones de la experiencia humana tienen un papel fundamental, especialmente en el aspecto cognitivo, las prácticas socioculturales y la dimensión ética y moral, es decir, la afectividad impacta en el conocimiento y el comportamiento moral, y, por tanto, en la dimensión evaluativa (Bortolan, 2017). En este sentido, propone que las experiencias afectivas serían evaluaciones sentidas, a través de las cuales juzgamos a personas, eventos o estados de cosas, como poseedores de un valor particular.

Del mismo modo, las propiedades axiológicas que se movilizan en la existencia se experimentan a través de los diferentes tipos de estados afectivos. Al respecto, Slaby (2016) puntualiza que la experiencia de una emoción implica, generalmente, la presencia de una inclinación a actuar de cierta manera, debido, principalmente, a los componentes motores asociados con las emociones. Por tanto, la afectividad movería a actuar de formas particulares, 
estrechamente relacionadas con las evaluaciones y la experiencia afectiva. Seyler (2016) señalaría una asociación entre afectividad e intencionalidad.

En este punto, es necesario detenerse a reflexionar acerca de cómo estamos concibiendo la afectividad. Al respecto, muchos autores coinciden en la complejidad de consensuar una definición, puesto que su contenido se encuentra en todas las experiencias del sujeto. No obstante, afectividad viene de la palabra afectación, del latín affectatio, y hace referencia a una impresión interior producida por factores externos o internos. Así, la afectividad da cuenta de una serie de procesos psíquicos, entre los cuales podemos identificar emociones, sentimientos y pasiones, manifestaciones propias del ser humano que anuncian la manera en que nuestro ser interior es afectado por todo lo que ocurre en su exposición al mundo. Llama la atención que para autores como Meylan (2017), incluso las intuiciones serían estados afectivos similares a las emociones, más que simples procesos perceptuales.

Por lo tanto, desde una perspectiva fenomenológica, la existencia cotidiana estaría impregnada de una multitud de experiencias afectivas, en que la mayor parte de las emociones, los estados de ánimo y los sentimientos están presentes para nosotros de forma próxima y enérgica. Desde aquí, la propia afectividad conlleva una fuerza que nos motiva e incluso nos obliga a perseguir ciertos objetivos. Estas experiencias significativas revelan ciertas características de las situaciones en las que participan los sujetos y, por tanto, pueden arrojar luz sobre la definición de situaciones y la información acerca de cómo actuar en ciertos contextos (Elpidorou y Freeman, 2015).

Dichas comprensiones nos llevan a deducir que la experiencia afectiva es una dimensión en la que es posible intervenir. Desde la perspectiva de Foucault (1990), se movilizan tecnologías que encauzan, producen y canalizan la experiencia. Por otra parte, Slaby (2016) señala que es posible conformar complejas estructuras de sentimientos, con el propósito expreso de facilitar la lealtad frente a modos de ser o de comportarse, que a su vez resultan conducentes a los objetivos de una determinada institución y/o empresa. Ilustrativamente, podríamos remitir a los vínculos afectivos que establecen con nińos y adultos los profesionales docentes que trabajan en las aulas hospitalarias. Estos vínculos que se entrelazan con un trabajo omnipresente y con recompensas espirituales son difíciles de definir desde la experiencia afectiva, puesto que carecen de un espacio para la evaluación crítica, debido a que, según Butler (2007), somos nuestros propios apegos afectivos.

Del mismo modo, desde la perspectiva fenomenológica, la agencia se constituye en un entramado cognitivo-afectivo, en un compromiso y respuesta corporal, cuyo resultado es que no solo los cerebros de los sujetos se movilizan 
en el aprendizaje, sino que también lo hace la sintonía corporal y afectiva con su entorno (Maiese, 2017), vislumbrando la afectividad como raíz del capital simbólico y motor de la constitución de la subjetividad (Ferme, 2009).

Así, desde las actuales discusiones respecto a la afectividad, podemos señalar que actualmente no es posible concebirla como una mera cuestión de procesos ligados al organismo, sino más bien como una susceptibilidad constituida por estructuras del entorno, contribuyendo con ello a que la experiencia afectiva pueda ser comprendida como una práctica cultural estructurada activamente desde el entorno por un agente o un grupo de agentes, con el fin de lograr objetivos relacionales y realizar cambios conducentes a un bien-estar.

Adicionalmente, otorgar al proceso el carácter de experiencia nos permite dar cuenta que en ella irrumpe el acontecer, la posibilidad, lo improbable e imposible, la extrañeza, la fluidez y la continuidad-discontinuidad que se configura desde un «materialismo de lo incorporal» (Contreras, 2012). En palabras de Bárcena, Larrosa y Mèlich (2006), daría cuenta de lo que nos acontece y singulariza, porque no responde a lo planificado. En suma, tal como lo seńala Trebisacce (2016), la experiencia es una invención epistémica que tiene como objetivo dar cuenta de aquello que resulta excedente, subsidiario o invisible a la ciencia legitimada como tal. Por tanto, la experiencia educativa implica que todo saber se localiza en un mundo de experiencias vividas. En que la labor docente se constituye en una existencia sensible de afecciones y afectos propios del mundo de la vida, en que lo vivido se orienta a la búsqueda de sentido (Jiménez y Vázquez, 2017).

\subsection{Un estudio sobre las concepciones}

Consideramos que reconocer las concepciones que fundamentan las acciones docentes puede contribuir a una pedagogía del tacto desde una ética responsable, que restaure nuestra humanidad y acoja la vulnerabilidad, contingencia, precariedad y finitud de la condición humana. Se comprende que las concepciones se encuentran ligadas a las actitudes, expectativas y creencias, y que todas ellas instituyen y constituyen las prácticas.

Estas prácticas que pueden ser entendidas en referencia tanto a las concepciones como a los significados que le atribuyen y que están enmarcados por dimensiones sociales, culturales históricas y políticas. Es decir, su principal característica sería su carácter eminentemente social, ya que se fundamentan e interrelacionan en espacios y situaciones culturalmente compartidas (Hidalgo y Murillo, 2017). En otras palabras, las concepciones se constituyen como organizadores implícitos, que en el caso de los docentes abarcan tanto sus 
conocimientos profesionales como sus creencias, las que se entrelazan con la experiencia profesional y objetivan en el contacto con la realidad escolar (Prieto y Contreras, 2008).

A partir de aquí, queda en evidencia que la preocupación por conocer las concepciones respecto a la afectividad tiene su razón en el deseo de relevar la experiencia afectiva en la acción educativa, en función de los escenarios formativos y sociales donde esta se configura, permitiendo comprender dicha experiencia en función del papel que cumple en el acto educativo mismo, el cual es eminentemente social, político, cultural, normativo y ético (Contreras y Ramírez, 2015). De esta manera, es posible recuperarla del ámbito a la que ha sido relegada como una mera entidad intrapsíquica, inherente al proceso individual.

Lo anterior resulta pertinente cuando la experiencia educativa adopta un carácter positivista, prescriptiva, taxonómica, normativa y reguladora, para alcanzar fines prácticos y utilitarios, o el legado de un bien moral y epistemológico de un mundo racional y lógico (Jiménez y Vázquez, 2017)

Este trabajo se justifica en la posibilidad que a partir de estos hallazgos se pueda comprender la importancia de abordar este aspecto en la formación inicial y continua de los docentes, constituyendo un desafío en términos epistemológicos, teóricos, metodológicos y didácticos, al considerar las particularidades de algunas modalidades educativas, como son las aulas hospitalarias.

Con todo, este artículo se plantea como objetivo principal describir las concepciones de afectividad que elaboran docentes que trabajan en un establecimiento educativo, ubicado al interior de un centro hospitalario, en la Región Metropolitana de Chile.

\section{Metodología}

Los resultados que se exponen a continuación corresponden a un estudio de carácter descriptivo (Cazau, 2006), en la medida que se investiga e intenta describir una situación actual, tanto en el ámbito de la educación como de las ciencias sociales, relacionado particularmente con la experiencia afectiva de docentes de aulas hospitalarias.

El diseño de esta investigación responde a un estudio de caso (Stake, 2007). Para este ejercicio, el "caso» lo constituye una escuela hospitalaria, situada en un hospital público de la Región Metropolitana de Chile. En este sentido, se abordan las concepciones como organizadores de las prácticas que los docentes atribuyen a su trabajo, desde un enfoque cualitativo (Flick, 2007). Este método permite analizar significados, vivencias y creencias que se 
presentan en los relatos de docentes, otorgando sentido a las experiencias que configuran su labor dentro de un hospital.

El establecimiento atiende, en promedio, a treinta niños con diagnóstico oncológico. Para efectos de esta investigación, fue escogido atendiendo a su disponibilidad para participar. En cuanto a los participantes, estos correspondieron al total del equipo docente, integrados por seis profesionales que realizan docencia (ver Tabla 1).

Tabla 1. Descripción de los participantes

\begin{tabular}{|c|c|c|c|c|c|c|}
\hline Entrevistado/a & Edad & Profesión & $\begin{array}{l}\text { Años de } \\
\text { estudio }\end{array}$ & $\begin{array}{c}\text { Años de } \\
\text { experiencia }\end{array}$ & $\begin{array}{l}\text { Años de } \\
\text { trabajo en } \\
\text { la escuela }\end{array}$ & $\begin{array}{c}\text { Función en } \\
\text { la escuela }\end{array}$ \\
\hline Entrevistado 1 & 24 & $\begin{array}{l}\text { Profesor de } \\
\text { Educación } \\
\text { General Básica }\end{array}$ & 5 años & 2 años $1 / 2$ & $\begin{array}{l}\text { Primer } \\
\text { año }\end{array}$ & $\begin{array}{l}\text { Profesor de } \\
\text { segundo } \\
\text { ciclo, de } \\
\text { quinto } \\
\text { al octavo } \\
\text { básico y } \\
\text { educación } \\
\text { media }\end{array}$ \\
\hline Entrevistada 2 & 27 & $\begin{array}{l}\text { Profesora de } \\
\text { Educación } \\
\text { General } \\
\text { Básica y } \\
\text { psicopedagoga }\end{array}$ & 5 años & 3 años & 1 año $1 / 2$ & $\begin{array}{l}\text { Profesora de } \\
\text { primer ciclo, } \\
\text { de primero } \\
\text { a cuarto } \\
\text { básico }\end{array}$ \\
\hline Entrevistada 3 & 43 & $\begin{array}{l}\text { Profesora de } \\
\text { Educación } \\
\text { General Básica } \\
\text { y licenciada en } \\
\text { educación }\end{array}$ & 5 años & 15 ańos & 11 ańos & $\begin{array}{l}\text { Directora y } \\
\text { docente de } \\
\text { la escuela }\end{array}$ \\
\hline Entrevistada 4 & 39 & $\begin{array}{l}\text { Educadora } \\
\text { diferencial } \\
\text { Educadora de } \\
\text { párvulos }\end{array}$ & $\begin{array}{l}5 \text { años de } \\
\text { diferencial } \\
3 \text { ańos } 1 / 2 \\
\text { de párvulo }\end{array}$ & 12 ańos & 12 años & $\begin{array}{l}\text { Educadora } \\
\text { de párvulos } \\
\text { y jefa de } \\
\text { UTP }\end{array}$ \\
\hline Entrevistada 5 & 34 & $\begin{array}{l}\text { Diseńadora } \\
\text { industrial } \\
\text { cursando } \\
\text { magíster en } \\
\text { Arte en la salud } \\
\text { y Arte-terapia }\end{array}$ & $\begin{array}{l}6 \text { años de } \\
\text { diseño } \\
2 \text { años de } \\
\text { magíster }\end{array}$ & 3 años & 3 años & $\begin{array}{l}\text { Profesora } \\
\text { de artes } \\
\text { plásticas }\end{array}$ \\
\hline Entrevistado 6 & 27 & $\begin{array}{l}\text { Educador } \\
\text { diferencial }\end{array}$ & 5 años & 5 años & 5 años & $\begin{array}{l}\text { Profesor en } \\
\text { atención } \\
\text { domiciliaria }\end{array}$ \\
\hline
\end{tabular}


Para la construcción de la información, se realizaron entrevistas individuales semiestructuradas (Flick, 2007). El registro de la información de las entrevistas fue grabado en formato de audio, con la finalidad de ser transcrito posteriormente. La pauta de entrevista se elaboró en base a las siguientes temáticas: definición de afectividad, acontecimientos que inciden en la afectividad, dimensiones que constituyen la afectividad, expresiones de la afectividad, expresión de la afectividad en las aulas hospitalarias, propuestas pedagógicas, rol docente, barreras y oportunidades del trabajo docente en aulas hospitalarias, afectividad e infancia, afectividad y enfermedad, entre otras.

Para el análisis de la información, se consideró un enfoque cualitativo con énfasis en la interpretación de los relatos, las respuestas y/o declaraciones de los docentes, en el marco de una organización conceptual que guio el análisis de la información construida (Stake, 2007). Específicamente, se opta por el análisis temático. Un método que permite identificar, organizar, analizar en detalle y reportar patrones a partir de una cuidadosa lectura y re-lectura de la información recogida, que, según Mieles, Tonon y Alvarado (2012), contempla las siguientes fases:

- Fase 1: Familiarización con la información, que consiste en la transcripción, lectura y relectura del material y anotación de ideas generales, buscando estructuras y significados.

- Fase 2: Generación de códigos iniciales, en que se organiza la información en grupos de un mismo significado codificando de manera inductiva y teórica la mayor cantidad de patrones en la información sin perder la perspectiva del contexto.

- Fase 3: Búsqueda de temas, es decir, un tema que capture algo importante de la información en relación con la pregunta de investigación.

- Fase 4: Revisión de temas, en que se realiza la re-codificación y el descubrimiento de nuevos temas.

- Fase 5: Definición y denominación de temas, incorporando la jerarquización

- Fase 6: Elaboración del meta-relato

Finalmente, dejamos constancia que se cumplió con los resguardos éticos considerando aspectos como confidencialidad y consentimiento informado de todos los participantes. 


\section{RESUlTADOS Y DisCUSIÓN}

En términos generales, los hallazgos que se desprenden de nuestra investigación apuntan a tres aspectos centrales, dos de ellos son similares a los expuestos en la investigación desarrollada por Bustos y Cornejo (2014). Para estos investigadores, los sentidos atribuidos al trabajo en aula hospitalaria se aprecian en dos áreas de significación. Por un lado, facilitar la reinserción de estudiantes en la escuela formal y por otro, contribuir a establecer relaciones de aula como un espacio de normalidad. El tercer hallazgo, que nace al alero de nuestra investigación, hace referencia a la concepción de responsabilidad de los docentes, en relación con contener y acompañar a los niños en el proceso de tratamiento de su enfermedad.

En cuanto a la vivencia de las dimensiones afectivas del trabajo, se encontraron los mismos núcleos de significado identificados por Bustos y Cornejo (2014), relacionados con la validación de las emociones, su vivencia extrema y oscilante, la intensidad de los vínculos afectivos establecidos y la valoración de los aspectos afectivos del trabajo como un privilegio.

A modo de ilustración, el siguiente fragmento contiene lo expuesto por una entrevistada que lleva once años trabajando en aula hospitalaria:

«Aquí, yo creo que es un lugar súper privilegiado en cuanto a la afectividad. El contacto que uno tiene con los niños es mucho más cercano, los niños son súper respetuosos. Ahora... es difícil a veces, es súper contradictorio, porque uno tiene que ser súper afectivo con sus alumnos por el proceso que están llevando y ellos, como nos ven que los acompańamos, son mucho más carińosos, son mucho más acogedores y mucho más receptivos a lo que nosotros les queremos entregar. Pero también por otro lado, hay niños que son mucho más retraídos, mucho más depresivos, que no se dejan mucho querer, o a veces no nos tienen confianza, pero son los menos» (E3).

Por consiguiente, creemos importante señalar que la categoría afectividad no estaría considerada como un fenómeno que es teorizado y recreado en el discurso de los docentes entrevistados, lo cual es considerado como un hallazgo de tipo general. Asimismo, se aprecia en sus relatos una débil consistencia y coherencia entre las diversas concepciones y nociones que constituyen su discurso.

Desde otro punto de vista, se señala que algunas de estas declaraciones contienen múltiples voces, percibiéndose como altamente pluralizadas, constatándose que en el discurso de un mismo participante convergen una serie de concepciones, que pasan de marcos descriptivos en torno a elementos constitutivos como las emociones y sentimientos, a marcos explicativos en función 
de aspectos biológicos o neurológicos. Asimismo, transitan desde concepciones alojadas en una dimensión individual a una relacional y viceversa.

En este sentido, es relevante destacar un fragmento del discurso de una entrevistada que lleva doce años trabajando en aula hospitalaria. Ilustra un discurso multifocal en que se pueden apreciar diversas tonalidades y contenidos.

«Si yo tengo una emoción... quizás negativa frente al hospital, mi afectividad va a disminuir. Quizás mi relación o el trabajo que yo ejecute en ese lugar no serán del todo beneficioso para la parte que la recibe. Esto, si yo voy temerosa o si mis emociones no las tengo controladas. Para eso, hay que tener carińo, amor... bueno, todas las emociones en general, desde la rabia, la tristeza, la gran gama que hay, porque uno pasa por muchas» (E4).

Una posible respuesta para lo descrito se encontraría en la línea que señala que la experiencia afectiva no pasa por el discurso, aunque si lo hace por la vida cotidiana, constituyéndose en una experiencia que excede al lenguaje (Martínez-Chaparro, 2010).

Abordadas algunas generalidades de los hallazgos de la investigación y comprendiendo que son un marco de referencia imprescindible para el ejercicio interpretativo, nos corresponde dar cuenta de las diversas concepciones que cohabitan en los discursos de los participantes.

En primer lugar, están con relación a cómo definen la afectividad los docentes, la cual anuncian en oraciones precedidas por el verbo «es». Un verbo indicativo, singular, en tercera persona, intransitivo. Este verbo es acompañado frecuentemente en los discursos por el adverbio y/o pronombre indefinido «algo»

«...la considero como algo muy ligado a lo emocional, que es muy distinto a lo mental o a lo físico" (E1).

"La afectividad para mi es algo que va desde que nacemos hasta que la persona muere, se entiende como algo que está en todas las etapas de nuestra vida» (E6). «... es algo que está presente siempre» (E1).

Se revela que la experiencia de la afectividad es concebida como un «algo», que, a pesar de su función como pronombre indefinido, posee límites y contornos que lo distinguen de otros fenómenos. Es decir, correspondería a un «algo» omnipresente y constituyente de la existencia. De este modo, remite a pensar en una señal indeterminada, haciendo referencia a aquello que no se puede nombrar o no se logra especificar.

En este sentido y desde la perspectiva de Cassirer (2016), la tarea primigenia de la conceptualización es particularizar la representación, es decir, atender a 
la exigencia de la universalidad del concepto, lo cual se asimila a un vehículo que permite alcanzar la verdadera meta que es la precisión. No obstante, en el «algo» seńalado por los/as entrevistadas se observa una situación distinta, ya que si bien en algunos fragmentos se aprecian afirmaciones y diferenciaciones - las cuales se acercarían a la precisión señalada por el autor-, en otros fragmentos se observa un ir y venir de imprecisiones sensibles, que parecen detenerse y encontrar algunos puntos de reposo.

Consideramos como puntos de reposo la asociación a la experiencia de cierta materialidad, que es posible de percibir y hasta cuantificar, pero, sobre todo, de controlar.

«....recibimos afectividad por parte de nuestros padres y después también nosotros vamos sintiendo un vínculo de apego" (E6).

«La afectividad uno la expresa y la demuestra en la medida que uno se sienta en confianza para poder hacerlo, en un lugar seguro para uno" (E2).

Así, recibir o no recibir afecto es una concepción que constantemente recorre los relatos de los docentes, remitiendo a una exterioridad más que a una experiencia de sí mismo. Es decir, no se estaría concibiendo como un estado de afectación en su relación con el mundo o en el mundo, sino que se aprecia, más bien, como una experiencia que se configura y emerge en las relaciones interpersonales y que es procesada y organizada desde lo individual, a partir de experiencias significativas.

«...me parece que es como las relaciones humanas que existen entre las personas» (E3).

Estas experiencias significativas estarían enmarcadas en todos los discursos, en mayor o menor medida, particularmente en la demostración de emociones y sentimientos positivos.

«La afectividad es toda demostración de cariño, ya sea física o emocional hacia alguien, todo lo que pueda sentir por alguien» (E2).

«Encuentro que hay nińos demasiado cariñosos y otros a los que les falta un poco demostrar su afectividad» (E6).

«Yo en el fondo... como que siempre he pensado que una de las cosas más importantes de dar carińo, es que el otro quiera recibirlo. Entonces, por ejemplo, a veces hay uno que es muy efusivo, y va y abraza a una alumna y ella no quiere, entonces trato de ir enseñando ese tipo de cosas, que en el fondo... como que uno tiene un espacio privado, que no puede llegar a cualquiera y molestarlo, como que siempre tiene que ser mutuo» (E2). 
Apreciamos en los discursos declaraciones asociadas a una evaluación sentida. En palabras de Bortolan (2017), la experiencia afectiva sería considerada como tal, en la medida que su propiedad axiológica emerja de estados afectivos relacionados con el apego y el amor. En este sentido, reconocemos que los discursos de los docentes conciben la experiencia afectiva como un proceso interactivo, que, no obstante, es padecido individualmente.

Desde aquí es posible establecer que la expresión de la afectividad es un tema central en los discursos de los docentes. Asimismo, descubrimos que, sin ser nombrado directamente, nuestros entrevistados hacen mención al cuerpo. Es decir, sus expresiones presentan algunas aseveraciones que identifican el sercorporal en-el-mundo y el cuerpo como modo de expresión.

De este modo, y desde el discurso de los docentes, la condición corpórea de la experiencia afectiva se expresa en gestos y acciones solo entre nińos. En otras palabras, el cuerpo de los niños tendría la condición de un cuerpo propio, vivido, sintiente: Un cuerpo que hace algo, que experimenta. Desde la perspectiva de Merleau-Ponty (1975), sería un cuerpo quiasmático, es decir, concebido como dualidad, en términos de sobre-posición, de mutua referencia entre experiencia afectiva y cuerpo.

«Bueno entre ellos también se expresan afectos, por el hecho de tener... por ejemplo, una niñita con silla de rueda necesita el estuche, y los nińos demuestran su carińo ayudando" (E2).

«Ellos también son carińosos, me abrazan, se acuerdan de cosas que hicimos la clase pasada, se acuerdan de uno, te prestan atención, te respetan y respetan la clase misma» (E5).

Sin embargo, cuando se hace referencia a su modo de expresión, los discursos aparecen más heterogéneos. Así, destacan aquellos en que el cuerpo ya no está presente y la expresión verbal aparece como el modo sintiente. En este sentido, nos recuerdan la tradición filosófica griega que enseña que los afectos se sitúan en oposición dialéctica a la razón. Específicamente, en la figura de Logos, quién «establece el orden del universo y la verdad oculta bajo las apariencias mundanas, prevaleciendo frente al caos de las pasiones introducido por pathos y eros» (D' Aubeterre, 2005).

En otros discursos se reconoce que la experiencia afectiva del cuerpo es concebida como intersubjetividad-intercorporalidad. A partir de aquí, la afectividad se configura como parte del mundo circundante, expresándose como una actuación en común de sujetos enlazados, social y culturalmente. Es decir, los otros son la aportación co-constituyente sin la cual no habría afectividad. 
«Yo trato de expresar mi afectividad prestándoles atención, echándoles tallas, hablado de sus cosas, mostrándoles interés en sus temas, estimulándolos a que hagan... en mi caso actividades artísticas. Como fomentándoles el amor por la actividad artística y destacándoles mucho lo bueno, más que lo malo» (E5).

«La afectividad se percibe por distintos sentidos y más que sentidos, también por la intuición, que es un tipo de percepción muy sutil, donde uno puede discernir entre distintas emociones y sentimientos. Se pueden también expresar o manifestar de distintas formas» (E1).

En este último relato se aprecia que la afectividad «se percibe por distintos sentidos», destacando el hecho del "sentir», lo que se encuentra directamente relacionado con el concepto de afectividad investigado, por cuanto la experiencia subjetiva de "sentirse a sí mismo", es decir, de sentirse afectado, tal como señala el participante, ayuda a discernir entre distintas emociones y sentimientos, dando cuenta de una experiencia de sí mismo.

Del mismo modo, la afectividad es concebida por todos los docentes, en mayor o menor grado, como una experiencia socioculturalmente mediada, debido a que se configura a partir de las condiciones de vida, tanto materiales como del entramado de relaciones en que el sujeto se afirma. En otras palabras, la afectividad se constituye en las situaciones y lugares, así como en el cuerpo que habita y es habitado.

"Hay que reconocer las emociones que tenemos todos frente a cualquier actividad humana o relaciones que se dan el aula, en el patio, en el hospital, o donde sea, las emociones que nos inundan a todos, y que nos permiten actuar y resolver según esas mismas emociones» (E4).

Tal como se desprende de los discursos de los participantes, es posible visualizar lo singular del acontecer educativo que se constituye en las aulas hospitalarias, donde la experiencia afectiva conforma una dimensión inherentemente social, en que hablar de afectividad es en última instancia, hablar de una afectividad colectiva.

"Creo que somos una institución muy carismática y que está en nuestros valores como institución entregar afecto a otros» (E3).

«Es una de las razones por las que los profesionales estamos acá, porque nos gusta, porque sentimos carińo y amor por este tipo de trabajo, y eso es súper necesario para que los nińos lo perciban desde el momento en que llegan y confíen en los profesionales que están a cargo de ellos. Que los vean como personas reales, cercanas, en las que pueden confiar y que saquen algo positivo de tu vida» (E1). 
«Pienso que somos una escuela bastante afectiva y muchas veces nos escapamos de los limites. Yo creo... creo que nuestro contexto hace que uno transgreda un poco los límites de la afectividad, ya que tenemos en ocasiones estudiantes que están complicados o tenemos en ocasiones estudiantes que fallecen y eso hace que uno se involucre y no solo con el estudiante, sino que también con su familia» (E6).

Los fragmentos anteriores confirmarían la idea de una afectividad colectiva, resaltando lo expresado por el entrevistado 6, quien señala la importancia del trabajo con la familia, sobre todo en el contexto hospitalario donde se viven experiencias de pérdida o dolor. En esta línea, retomamos lo señalado por Mèlich (2003), quién plantea la relevancia de educar en la fragilidad y finitud humana, específicamente a partir de la vulnerabilidad de los estudiantes. Asimismo, se realzan también los principios de la pedagogía del tacto que implementan los docentes, destacando los lazos que se proyectan entre los actores. A su vez, se apela a que las relaciones creadas en el contexto hospitalario son una de las principales razones por las cuales los docentes se sienten enamorados de su labor y aspiran a que los estudiantes confíen y se conecten con ellos.

\section{Conclusiones}

Esta investigación y sus hallazgos permiten reflexionar acerca del concepto de afectividad. Concepto que, mediante el giro afectivo, da cuenta de un fenómeno que arrastra una sedimentación ideológica muy variada, fruto de incrustaciones históricas, políticas, culturales y territoriales. En el caso de los docentes que trabajan en el aula hospitalaria, remite particularmente a una determinada concepción de afectividad, que cobra forma y sentido en su asociación con la propiedad axiológica que emerge de los estados afectivos relacionados con el amor. Por ello, relevar esta asociación en y desde los discursos de los docentes implica asumir, de acuerdo a Parker (1992), que estas afirmaciones construyen un objeto, una realidad y, finalmente, una práctica docente. Por tanto, dicha premisa demanda comprender que restringir la afectividad a determinados estados afectivos significaría limitar la condición per se, propiciando una pedagogía que se aleja de lo humano. Entendiendo que humanizar es aceptar y habitar en un mundo que afecta.

Lo mencionado anteriormente, resulta relevante si consideramos lo que plantea Slaby (2016), en el sentido que la afectividad configura patrones, relaciones, disposiciones y significados, los que a su vez instituyen nuevas prácticas. 
En consecuencia, consideramos que el estudio de las concepciones de la afectividad va más allá de exponer su contenido, tal como lo hemos hecho en este artículo. Debería, además, indagar acerca de su naturaleza, de manera que sea posible comprender cuestiones de fondo, por ejemplo, de qué forma se instituyen las prácticas y cuáles son los mecanismos de retorno a lo humano en la experiencia afectiva, en términos amplios y fenomenológicos.

En otras palabras, se aprecia que la afectividad es descrita por los docentes entrevistados como un medio para intensificar la vida, de acuerdo a un sentido más bien instrumental. Así y atendiendo al riesgo que implica que la afectividad se instale en un espacio protegido, surge el eco romántico, conservador e instrumentalizado de sitiar la afectividad, lo cual se aprecia en los discursos, en la ausencia de ideas relacionadas con el cuerpo, la afectación, la subjetividad, el carácter político, normalizador, social e histórico, que lleva al error de reservar la experiencia afectiva para el amor, conservándola en una arcaica caja de tesoros de la humanidad. Si bien, dicho tesoro es gestionado y manipulado de acuerdo a ciertos intereses, entre ellos, un sistema productivo releva como su más precioso recurso, la experiencia afectiva.

Por lo tanto, a pesar que en las concepciones se aprecia una dimensión relacional, esta cohabita subordinada a un rasgo solipsista de los sentimientos, emociones e intuiciones. En otras palabras, se visualiza la experiencia de sí mismo como una coexistencia, como la presencia de una radical alteridad. Sin embargo, esta forma de concebir la experiencia afectiva, por parte de los docentes, permite avanzar en develar una representación particular de dicha experiencia.

En este sentido, es relevante plantear que el cuerpo tiene una presencia muy difusa en las concepciones de los docentes, diluyéndose en sus discursos. Situación que prende una luz de alarma en el contexto educativo de un aula hospitalaria, ya que, frente al dolor de una enfermedad, los cuerpos adquieren una presencia insoslayable. A partir de aquí, las conclusiones desafían a los docentes a reconocer la revelación de la corporeidad como unidad de la experiencia afectiva y vital, humanizando la categoría de cuerpo y superando definiciones que lo asimilan a objeto, cosa sensible o materia inerte. De esta manera, se daría cabida a la promoción de existencias que se afirman en el cuerpo, transformándose, por tanto, en pura afectividad, coincidiendo con lo planteado por Ballén (2012) en cuanto a que «la revelación de la vida en el cuerpo tiene lugar en la afectividad» (p. 132). 


\section{REFERENCIAS BIBLIOGRÁFICAS}

Arredondo, T. (2014). La pedagogía hospitalaria en Chile. Santiago: Mineduc.

Ascorra, P. (2009). El gerenciamiento de los estados de ánimo: Estudio de caso en una organización chilena. Época, 20, 21-32.

Ballén, J. (2012). El lenguaje fenomenológico de la afectividad en Michel Henry: la reconstrucción de una gramática de la subjetividad «a flor de piel». Acta Fenomenológica Latinoamericana, 4, 115-147.

Bárcena, F., Larrosa, J. y Mèlich, J. (2006). Pensar la educación desde la experiencia. Revista Portuguesa de Pedagogía, 1, 233-259.

Bortolan, A. (2017). Affectivity and moral experience: an extended phenomenological account. Phenomenology and the Cognitive Sciences, 16, 471-490. https://doi.org/10.1007/s11097-016-9468-9

Bustos, C. y Cornejo, R. (2014). Sentidos del trabajo en docentes de aulas hospitalarias: las emociones y el presente como pilares del proceso de trabajo. Psicoperspectivas, 2(13), 186-197. https://doi.org/10.5027/ psicoperspectivas-Vol13-Issue2-fulltext-365

Butler, J. (2007). El género en disputa. Barcelona: Paidós Ibérica.

Carabantes, E. y Contreras, S. (2020). Educación humanizada para una democracia humanamente democrática. Revista Educación, 44(1). https://doi. org/10.15517/revedu.v44i1.37637

Cassirer, E. (2016) Filosofía de las formas simbólicas I. México: FCE.

Cazau, P. (2006). Introducción a la investigación en Ciencias Sociales. Buenos Aires: s/e.

Contreras, S. (2012). Pedagogía vulnerable y diálogo de saberes. Revista Integra Educativa, 3(5), 77-94.

Contreras, S. y Ramírez, M. (2014). Descolonizar la educación desde la crianza. Revista Electrónica Educare, 18(2), 297-309. https://doi.org/10.15359/ ree.18-2.15

Contreras, S. y Ramírez, M. (2015). ¿Es posible pensar en una pedagogía melancólica? En-claves del pensamiento, 9(17), 157-175.

D' Aubeterre, L. (2005). La construcción cultural de los afectos en Ciudad Guayana: un estudio psicosocial discursivo sobre los procesos de elaboración de narrativas y prácticas afectivas urbanas. Espacio Abierto, 14(2), 279-304.

Elpidorou, A., y Freeman, L. (2015) Affectivity in Heidegger I: Moods and Emotions in Being and Time. Philosophy Compass, 10, 661-671. https:// doi.org/10.1111/phc3.12236 
Ferme, F. (2009). Apuntes sobre la subjetividad y la afectividad: el proyecto de autonomía y la dimensión prerreflexiva de las prácticas. En I Congreso Internacional de Investigación y Práctica Profesional en Psicología. XVI Jornadas de Investigación. Quinto Encuentro de Investigadores en Psicología del Mercosur. Facultad de Psicología, Universidad de Buenos Aires.

Flick, U. (2007). Introducción a la investigación cualitativa. Madrid: Morata y Fundación Paideia Galiza.

Foucault, M. (1990). Tecnologías del yo y otros textos afines. Barcelona: Paidós Ibérica.

Freeman, L. y Elpidorou, A. (2015) Affectivity in Heidegger II: Temporality, Boredom, and Beyond. Philosophy Compass, 10, 672-684. https://doi. org/10.1111/phc3.12273

Garrido, V. (2016). La resiliencia: una intervención educativa en pedagogía hospitalaria. Revista de Educación Inclusiva, 9(1), 79-89

Heidegger, M. (1974). Ser y tiempo. Ciudad de México: FCE.

Hidalgo, N. y Murillo, F. (2017). Las concepciones sobre el proceso de evaluación del aprendizaje de los estudiantes. Revista Iberoamericana sobre Calidad, Eficacia y Cambio en Educación, 15(1), 107-128. https://doi. org/10.15366/reice2017.15.1.007

Jiménez, M. y Vázquez, A. (2017). Lo educativo como experiencia fenomenológica. Praxis \& Saber, 8(18), 253-268. https://doi.org/10.19053/22160159. v8.n18.2017.7243

Maiese, M. (2017). Transformative Learning, Enactivism, and Affectivity. Studies in Philosophy and Education, 36, 197-216. https://doi.org/10.1007/ s11217-015-9506-z

Martínez-Chaparro, Á. (2010). Apuntes sobre la noción de afectividad colectiva. Pensando Psicología, 6(10), 152-157. https://doi.org/10.1692/issn.1900-3099

Mèlich, J. (2003). Finitud y contingencia. En K. González (Comp.), Hilos y laberintos: irrupciones pedagógicas (pp. 100-120) Madrid: Miño y Dávila.

Merleau-Ponty, M. (1975). Fenomenología de la percepción. Barcelona: Península.

Meylan, A. (2017). Rationality and affectivity of the intuitions. Philosophiques, 44, 31-47. https://doi.org/10.7202/1040326ar

Mieles, M., Tonon, G. y Alvarado, S. (2012). Investigación cualitativa: el análisis temático para el tratamiento de la información desde el enfoque de la fenomenología social. Universitas Humanistica, 74, 195-225.

Ministerio de Educación (2003). Escuelas y aulas hospitalarias: programa de educación especial. Santiago: Mineduc.

Molina, M. (2017). ¿Qué es la pedagogía hospitalaria? Barcelona: Facultad de Educación, Universidad de Barcelona. 
Nunes, C. (2019). Formación y trabajo docente: cuestiones contemporáneas. Revista Exitus, 9(3), 19-32. https://doi.org/10.24065/2237-9460.2019v9n3ID917

Núñez, L., Núñez, M. e Irisarri, J. (2017). Afectividad normativa como fundamento de la autoridad doméstica en la sociedad digital. Revista Latina de Comunicación Social, 72, 331-348. https://doi.org/10.4185/ RLCS-2017-1168

Orealc/Unesco (2007). Aulas hospitalarias. Reflexiones de la VIII Jornada sobre Pedagogía Hospitalaria. Santiago: Libe Narvarte.

Organización de las Naciones Unidas (2015). Declaración Universal de Derechos Humanos (edición ilustrada). Nueva York: Naciones Unidas.

Parker, I. (1992). Discourse Dynamics: Critical Analysis for Social and Individual Psychology. Londres: Routledge

Prieto, M. y Contreras, G. (2008). Las concepciones que orientan las prácticas evaluativas de los profesores: un problema a develar. Estudios pedagógicos, 34(2), 245-262. https://doi.org/10.4067/S0718-07052008000200015

Red Latinoamericana y del Caribe (2009). Un gran logro en beneficio de los niños: Declaración de los Derechos del Niño, la Niña o Joven Hospitalizado o en Tratamiento de Latinoamérica y el Caribe en el Ámbito de la Educación. Río de Janeiro: Redlaceh.

Reyes-Laredo, F., Martín-Rubio, M. y Daza-Navarro, P. (2018). El papel del docente en un aula hospitalaria de oncología pediátrica. Revista Andina de Educación 1, 3-11. https://doi.org/10.32719/26312816.2018.1.1

Sánchez, A. y López, D. (2016). Evaluación de la respuesta educativa al alumnado de aulas hospitalarias en la provincia de Almería. Revista Española de Discapacidad, 4(1), 83-96. https://doi.org/10.5569/2340-5104

Seyler, F. (2016). Renaud Barbaras and Michel Henry: A Contemporary Debate on the Status of Affectivity and a Farewell to the «Flesh of the World». The Journal of Speculative Philosophy, 30, 395-403. https://doi. org/10.5325/jspecphil.30.3.0395

Slaby, J. (2016). Mind Invasion: Situated Affectivity and the Corporate Life Hack. Hypothesis and theory article. Frontiers in Psychology. 8, 1-13. https://doi. org/10.3389/fpsyg.2016.00266

Soto, R., Mera, J., Nuñez, C., Sisto, V. y Fardella, C. (2016). Between the effectivity and affectivity: new teachers in times of new public management. Journal Athenea Digital, 16, 3-19. https://doi.org/10.5565/rev/athenea.1528

Stake, R. (2007). Investigación con estudio de casos. Madrid: Morata.

Trebisacce, C. (2016). Una historia crítica del concepto de experiencia de la epistemología feminista. Cinta Moebio, 57, 285-295. https://doi.org/10.4067/ S0717-554X2016000300004 